\title{
Low rates of hyponatraemia in patients with diabetes treated with variable rate intravenous insulin infusion (VRIII)
}

\author{
ZEENAT BANU, ${ }^{1}$ DANIEL FLANAGAN²
}

\begin{abstract}
Aims: To investigate the risk of developing hyponatraemia or hypoglycaemia in individuals requiring variable rate intravenous insulin infusion (VRIII) using a protocol of variable rate insulin infused with $10 \%$ dextrose and $20 \mathrm{mmol} / \mathrm{L}$ potassium chloride.

Methods: A prospective study was performed of $\mathbf{5 0}$ consecutive medical and surgical patients requiring VRIII. Sodium, potassium and glucose were measured before, during and after VRIII use.

Results: Medical patients had significantly higher glucose values prior to VRIII. Glucose fell significantly in this group. Sodium concentrations were marginally low prior to commencing VRIII in seven medical and six surgical patients. One patient had significant hyponatraemia prior to VRIII (114 $\mathrm{mmol} / \mathrm{L}$ ) and this improved to $118 \mathrm{mmol} / \mathrm{L}$ during the VRIII. Overall there were no significant changes in sodium or potassium during VRIII. There were no significant differences in sodium or potassium between the two groups at any point.

Conclusion: There was a low incidence of clinically significant hyponatraemia and hypokalaemia associated with a commonly used intravenous insulin regimen that combines insulin with $10 \%$ dextrose.

Br J Diabetes 2019;19:45-48
\end{abstract}

Key words: variable rate intravenous insulin infusion,

hypoglycaemia, hyponatraemia, hypokalaemia

\section{Introduction}

Variable rate intravenous insulin infusions (VRIII) are commonly used to improve and maintain glucose control in acutely unwell patients.

Specialist Registrar, Department of Endocrinology, Derriford Hospital, Plymouth, UK

2 Consultant, Department of Endocrinology, Derriford Hospital, Plymouth, UK

Address for correspondence: Dr Daniel Flanagan

Department of Endocrinology, Level 9, University Hospital Plymouth,

Plymouth PL6 8DH, UK

Tel: +44 (0)1752 439696

E-mail: danielflanagan@nhs.net

https://doi.org/10.15277/bjd.2019.206
Consensus documents describe best practice for inpatient glycaemia and the use of VRIII. ${ }^{1-5}$ The most appropriate intravenous fluid to administer alongside the insulin is controversial. Few published papers have examined the metabolic changes associated with VRIII use, although dextrose infusion is implicated as a risk for inhospital hyponatraemia. ${ }^{6}, 7$ There is concern that dextrose infusion alongside insulin can achieve glycaemic control but may lead to clinically significant hyponatraemia.

There is limited evidence to demonstrate the superiority of any specific fluid regimen for VRIII. Until further clinical data are available, the UK guidance recommends $0.45 \%$ sodium chloride with $5 \%$ dextrose with additional potassium chloride (either $0.15 \%$ or $0.3 \%),{ }^{1}$ but this fluid is not available in all centres. Guidelines accept that dextrose alone may be a reasonable alternative infusion fluid.

The aim of this study was therefore to prospectively follow a cohort of medical and surgical patients treated with a VRIII protocol based on the national guidance but using 10\% dextrose as the infusion fluid running alongside intravenous insulin.

\section{Methods}

We prospectively studied 50 consecutive patients treated with VRIII admitted to University Hospital Plymouth, UK. Patients were not included if they were in intensive care or high dependency areas. Patients were excluded if they had an estimated glomerular filtration rate of $<15 \mathrm{~mL} / \mathrm{min}$ as this group were managed directly by the renal team. Patients requiring parenteral feeding were not included in the study as these patients are monitored daily by the nutrition team. Separate groups of medical and surgical patients were studied.

A VRIII was recommended for all perioperative patients with diabetes where the expected fasting time would result in two consecutive meals being missed, irrespective of whether they were treated with tablets or insulin. VRIII was recommended for a similar group of medical patients with prolonged fasting or cases of unstable diabetes where adjustment or use of other diabetes treatment regimens was thought to be inappropriate.

Insulin was infused at 0.5-6 units/hour. If blood glucose was $<15 \mathrm{mmol} / \mathrm{L}$, a fixed rate of $500 \mathrm{~mL} 10 \%$ dextrose with $10 \mathrm{mmol}$ potassium chloride at $1 \mathrm{~mL} / \mathrm{kg} /$ hour with a maximum rate of 100 $\mathrm{mL} /$ hour was administered. If blood glucose was $>15 \mathrm{mmol} / \mathrm{L}$, the dextrose infusion was substituted with $1000 \mathrm{~mL} 0.9 \%$ saline with $20 \mathrm{mmol} / \mathrm{L}$ potassium chloride at a rate of $1 \mathrm{~mL} / \mathrm{kg} / \mathrm{hour}$ with a maximum rate of $100 \mathrm{~mL} /$ hour. The target glucose range at the time of the study was 6-14 mmol/L. 
The VRIII protocol recommends hourly glucose measurements initially, then 2-hourly if glucose is stable. Blood electrolyte monitoring was recommended 12-hourly with a specific warning about vigilance for hyponatraemia or hypokalaemia. The protocol mandated that additional intravenous fluids to meet the patients' fluid and electrolyte needs $(0.9 \%$ normal saline or compound sodium lactate) be administered via volumetric pump. The diabetes specialist team is available for advice for all patients requiring VRIII but, as this was an observational study, no additional advice beyond normal standard of care was provided. We have included biochemistry prior to VRIII use, within the final 24 hours of the infusion, then 24-48 hours after the infusion and a final measurement more than 48 hours after the VRIII was stopped. The capillary glucose prior to starting the VRIII, mean capillary glucose from 6 hours after commencement of VRIII and mean capillary glucose within 6 hours of stopping the VRIII were recorded. Whether diabetes specialist input had been provided prior to commencement of VRIII or during use of VRIII was also recorded. If the patient was normally using a basal analogue insulin, this was continued alongside the intravenous insulin.

Repeated measures ANOVA was used to analyse changes in electrolytes with time. Equal variance was assumed. All data were analysed using MS Excel and SPSS Version 21 (IBM Inc, USA).

\section{Results}

The baseline characteristics of the medical and surgical patients are shown in Table 1.There was no significant difference in age between the two groups. As might be expected, glucose control as measured by $\mathrm{HbA}_{1 \mathrm{c}}$ was less good in the medical group compared with the surgical (mostly elective) group. The surgical group received more diabetes specialist input (a potential confounder). The duration of insulin infusion was significantly longer for surgical patients than for medical patients, varying from 8 hours to 2.7 days in medical patients and from 6 hours to 17 days in surgical patients.

Figure 1 compares glucose, sodium and potassium values before and over the course of the VRIII. Glucose was significantly higher in the medical group than the surgical group before commencement of the VRIII (mean (SD) 19.5 (8.7) mmol/L vs. 13.3 (7.5) $\mathrm{mmol} / \mathrm{L}$; $p=0.009$ ). Glucose fell significantly in the medical group during the infusion from 19.5 (8.7) $\mathrm{mmol} / \mathrm{L}$ to $13.9(5.2) \mathrm{mmol} / \mathrm{L}(\mathrm{p}=0.002)$. Having started at a lower level, glucose did not change significantly during the VRIII in the surgical group.

Mean sodium concentrations were $135 \mathrm{mmol} / \mathrm{L}$ in both groups before commencement of the VRIII and did not change significantly during or after the VRIII. Sodium levels at baseline were low $(<133$ $\mathrm{mmol} / \mathrm{L}$ ) in 13 patients (7 medical and 6 surgical). During the VRIII, seven patients remained hyponatraemic (3 medical, 4 surgical). One medical patient who had a normal serum sodium at baseline became hyponatraemic (sodium $131 \mathrm{mmol} / \mathrm{L}$ ). This patient had poor glucose control during the VRIII (mean glucose $18.8 \mathrm{mmol} / \mathrm{L}$ ). Twenty-four hours after VRIII, nine patients were hyponatraemic (3 medical and 6 surgical). Two surgical patients became hyponatraemic with normal serum sodium at baseline. Forty-eight hours after VRIII, seven patients ( 2 medical and 5 surgical) were found to

Table 1 Baseline characteristics and biochemical profile of patients on variable rate intravenous insulin infusions (VRIII)

\begin{tabular}{|c|c|c|c|}
\hline Baseline characteristics & Medical patients $(n=24)$ & Surgical patients $(n=26)$ & $P$ value for difference \\
\hline Gender male $(n)$ & 17 & 18 & NS \\
\hline Type 1 & 7 & 3 & \\
\hline Type 2 & 15 & 23 & \\
\hline Mean insulin rate on VRIII (units/hour) & 2 & 1 & $<0.001$ \\
\hline $\mathrm{HbA}_{1 \mathrm{c}}(\%, \mathrm{mmol} / \mathrm{mol})$ & $9,75.2 \pm 26.4$ & $8.1,65.2 \pm 20.2$ & 0.051 \\
\hline \multicolumn{4}{|l|}{ Treatment } \\
\hline OHA & 5 & 7 & \\
\hline $\mathrm{OHA}+$ insulin & 3 & 9 & \\
\hline DSN input & 14 & 24 & \\
\hline Mean (SD) length of stay (days) & $12.5 \pm 13.2$ & $18.6 \pm 28.4$ & 0.326 \\
\hline \multicolumn{4}{|l|}{ Hypoglycaemic episodes during VRIII } \\
\hline Major & 0 & 0 & \\
\hline Minor & 3 & 1 & \\
\hline
\end{tabular}


Figure 1. Mean (SD) sodium (A), potassium (B) and glucose (C) before, during and after the use of variable rate intravenous insulin infusion for a group of medical and surgical patients requiring insulin therapy. There are no significant differences in sodium or potassium values. Mean glucose fell significantly in the medical group

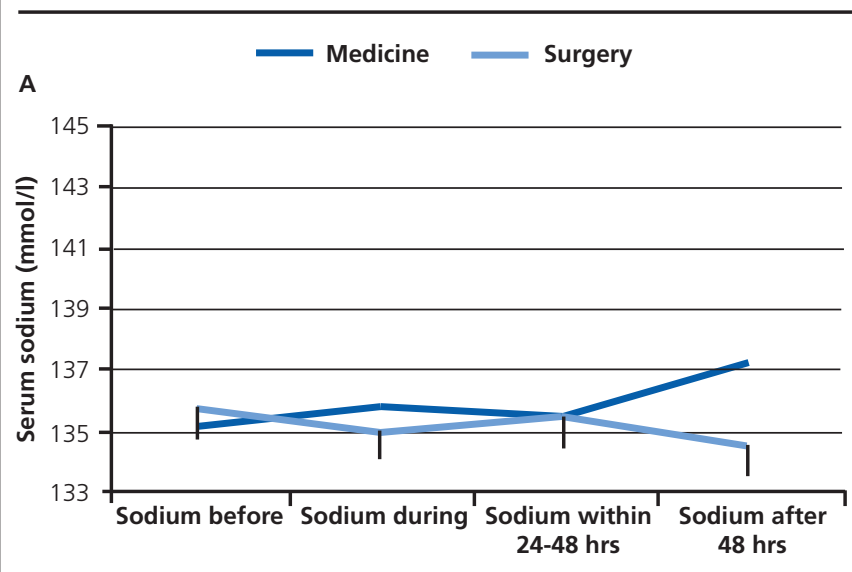

B
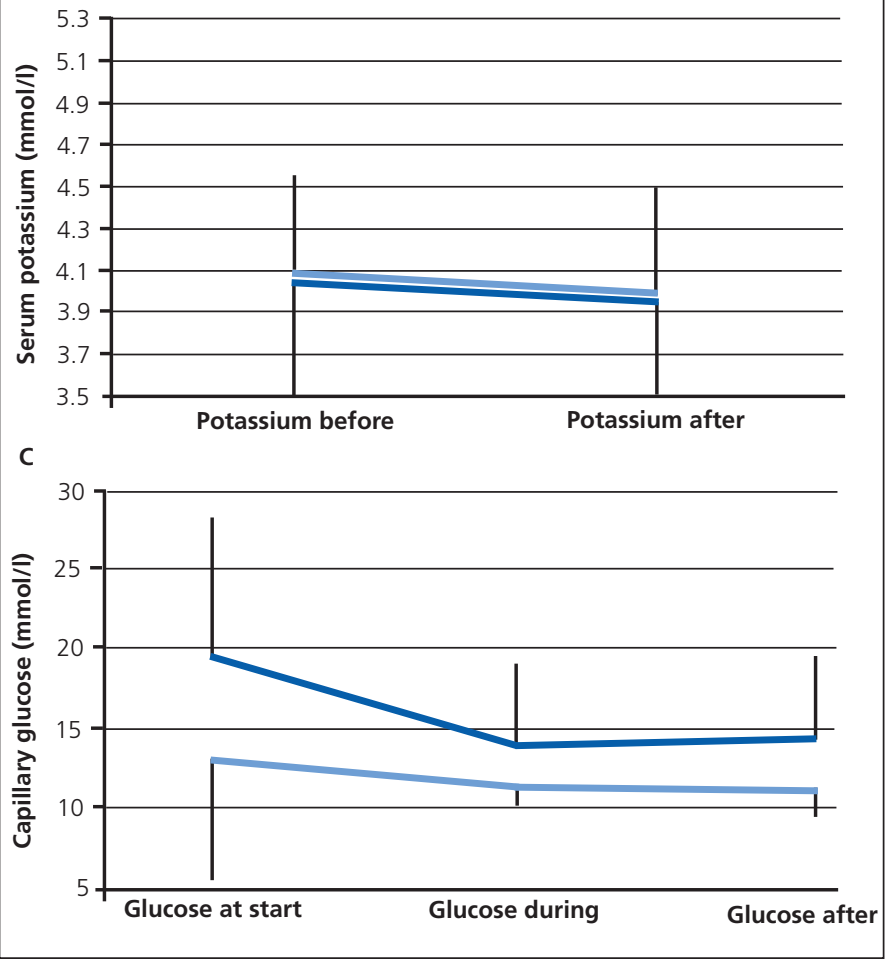

be hyponatraemic, of which two surgical patients had normal serum sodium at baseline. There was one case of clinically significant hyponatraemia (sodium $114 \mathrm{mmol} / \mathrm{L}$ ) prior to infusion and 118 $\mathrm{mmol} / \mathrm{L}$ during the infusion. This individual had a diagnosis of syndrome of inappropriate antidiuretic hormone secretion. Possibly the use of the standard VRIII was inappropriate in this case.

There was no significant difference in potassium concentrations between the medical and surgical groups before commencement of the VRIII and no significant change during the VRIII. Prior to VRIII, serum potassium was high in four patients ( 3 medical, 1 surgical).
After VRIII, one medical patient became normokalaemic and the remainder remained hyperkalaemic. Four patients (3 medical and 1 surgical) developed minor hypoglycaemic episodes during VRIII. There were no cases of clinically significant fluid overload during the time of VRIII use. Measured fluid balance was within acceptable clinical limits for all patients during the VRIII period. Four medical patients had additional $0.9 \%$ normal saline infusions during VRIII. Four surgical patients had additional $0.9 \%$ normal saline and five had additional compound sodium lactate. There was no difference in serum sodium concentrations between those patients who did and those who did not have additional intravenous sodium.

\section{Discussion}

This study shows that using a VRIII in combination with $10 \%$ dextrose, with supplementation of electrolytes as appropriate, results in a low rate of mild hyponatraemia and no cases of severe hyponatraemia.

VRIII is a commonly used tool to achieve normoglycaemia in hospital patients. Most acute hospitals will have guidelines for its use, but there is a wide variation in the indications for use, rate of fluid infusion, duration of use and indications to step down to other glucose-lowering therapies. In addition, there is variation in the infusion fluid that is used alongside the insulin. Audits of practice including the National Diabetes Inpatient Audit ${ }^{8}$ have shown that VRIII is often used when not indicated, its duration is unnecessarily prolonged and the step down to other glucose-lowering medication is often not practised safely.

Two recent documents produced by the Joint British Diabetes Societies Inpatient Group (JBDS) describe best practice for the use of VRIII in both medical and surgical patients. ${ }^{1,3}$ The documents detail the controversies in choice of infusate and acknowledge that there are few published data looking at biochemical control during VRIII. In the absence of specific information and extrapolating from other sources, the JBDS suggest $0.45 \%$ sodium chloride with $5 \%$ glucose with additional potassium chloride (either $0.15 \%$ or $0.3 \%$ ). This is currently the practice in many paediatric units. ${ }^{9}$ Before switching to what would be a novel fluid for our hospital, we decided to prospectively study biochemical control using our current protocol. Our current protocol is otherwise in agreement with JBDS guidance but uses 10\% dextrose as the infusion fluid.

As would be expected in a group of acutely unwell patients, there were fluctuations in serum sodium over the course of the study period, but we have not shown clinically significant hyponatraemia. After VRIII there was a non-significant trend towards lower serum sodium in the surgical group. The fall was from $135 \mathrm{mmol} / \mathrm{L}$ to 134 $\mathrm{mmol} / \mathrm{L}$, which is not clinically relevant. In this study we have not documented what happens to serum sodium in the days following discontinuation of VRIII. High blood glucose readings at the beginning of the infusion may have contributed to low serum sodium at that point, but sodium levels improved once better glycaemic control was achieved. We saw improvements in glucose control as one would expect over the course of the study and a relatively low rate of hypoglycaemic events. Potassium did not change significantly over the course of the study. Post-surgical hyponatraemia is a common problem even without VRIII use. This has multiple aetiologies including the physiological response to surgical stress and lack of 


\section{Key messages}

- Variable rate intravenous insulin infusions provide a safe way of maintaining glucose control in individuals who are unwell and require insulin

- Without careful monitoring there is the potential for harm as a consequence of abnormal glucose or biochemistry. There are particular concerns about potential low serum sodium or potassium levels

- A commonly used infusion regimen includes the use of insulin with $10 \%$ dextrose

- In our real-life study of the use of this regimen there was a low incidence of significant hyponatraemia or hypokalaemia, although monitoring remains suboptimal

attention to detail in fluid balance. ${ }^{10,11}$ It is perhaps not surprising to see a small fall in serum sodium in this group.

There are few published data to directly compare with this study. Papers have reported the association of the use of dextrose infusion with hyponatraemia as part of larger studies of the causes of hyponatraemia in hospitals, but details of the infusions used are not given. $6,11,12$

We have not reported details of other intravenous fluids used before the period of VRIII. While this may be considered a weakness of the study, the clinical question was whether this specific VRIII results in significant hyponatraemia. Details of other fluids would have been important if we had shown significant hyponatraemia but, as we have not, these details are less relevant.

Patients with hyponatraemia prior to commencement of VRIII were investigated appropriately. In a number of cases hyperglycaemia prior to commencing VRIII was a significant factor.

A weakness of this study is the relatively small number of patients but, even with this number, there were no trends towards falling serum sodium over the course of the infusion. A further weakness is the level of glucose control achieved during the VRIII. It will be noted that, although the mean glucose levels fall within the recommended range, the SD shows that a significant proportion lie above the normal range. This is partly due to the frequency of glucose testing and dextrose infusion adjustment, but this was not documented in this study. Although disappointing, this is comparable with levels of glucose control achieved nationally using the VRIII and perhaps contributes to the low rates of hypoglycaemia.

The protocol used required two measures of biochemistry every day for individuals using a VRIII, but this frequency was not achieved for 10 medical patients and 11 surgical patients. This could potentially lead to an underestimate of rates of biochemical disturbance.

We cautiously conclude that clinically relevant hyponatraemia appears to be an unusual problem in individuals using VRIII with insulin and $10 \%$ dextrose. Although we need to be cautious in interpreting a relatively small sample, this does suggest that a prospective comparative study with any new fluid regimen with careful measurement of electrolytes would be helpful. This should be performed before changing national recommendations.

\section{Conflict of interest: None \\ Funding: None}

\section{References}

1. The Joint British Diabetes Societies for Inpatient Care. The use of variable rate intravenous insulin infusion (VRIII) in medical inpatients. https://www.diabetes.org.uk/resources-s3/2017-09/Use\%20of\%20variable\%20rate\%20intravenous\%20insulin\%20infusion\%20in\%20medical\%20inpatients_0.pdf

2. The Association of Anaesthetists of Great Britain \& Ireland. Peri-operative management of the surgical patient with diabetes 2015. https://www.aagbi.org/sites/default/files/Diabetes\%20FINAL\%20published\%20in\%20Anaesthesia\%20Sept\%2015\%20with\%20covers\%20 for\%20online[1].pdf

3. The Joint British Diabetes Societies for Inpatient Care. Management of adults with diabetes undergoing surgery and elective procedures: improving standards. https://www.diabetes.org.uk/resources-s3/201709/Surgical\%20guidelines \%202015\%20-\%20full\%20FINAL \%20 amended\%20Mar\%202016_0.pdf

4. National Institute for Health and Care Excellence. Intravenous fluids for adults in hospital. NICE guideline [CG174]. London, 2013. https://www.nice.org.uk/guidance/cg174

5. Moghissi ES, Korytkowski MT, DiNardo M, et al. American Association of Clinical Endocrinologists and American Diabetes Association consensus statement on inpatient glycemic control. Diabetes Care 2009; 32:1119-31. https://doi.org/10.2337/dc09-9029

6. Harris B, Schopflin C, Khaghani C, Edwards M; Collaborators from the Southcoast Perioperative Audit and Research Collaboration (SPARC). Perioperative intravenous fluid prescribing: a multi-centre audit. Perioper Med (Lond) 2015;4:15. https://doi.org/10.1186/s13741-015-0025-9

7. Hoorn EJ, Lindemans J, Zietse R. Development of severe hyponatraemia in hospitalised patients: treatment related risk factors and inadequate management. Nephrol Dial Transplant 2006;21:70-6. https://doi.org/ 10.1093/ndt/gfio82

8. National Diabetes Inpatient Audit (NaDIA) 2015. http://content digital.nhs.uk/catalogue/PUB20206/nati-diab-inp-audi-15-nat-rep.pdf

9. ChizoAgwa J, Edge JA, Drew JH, et al. Association of Children's Diabetes Clinicians: Guidelines on the care of children under 18yrs of age with diabetes mellitus undergoing surgery. Version 1, October 2013. http://www.a-c-d-c.org/wp-content/uploads/2012/08/Care-of-childrenunder-18-years-with-Diabetes-Mellitus-undergoing-Surgery-1.pdf

10. Katz MA. Hyperglycemia-induced hyponatremia - calculation of expected serum sodium depression. N Engl J Med 1973;289:843-4.

11. Liamis G, Tsimihodimos V, Elisaf M. Hyponatremia in diabetes mellitus: clues to diagnosis and treatment. J Diabetes Metab 2015;6:1000560. https://doi/org/10.4172/2155-6156.1000560

12. Smiley $D$, Rhee $M$, Peng L, et al. Safety and efficacy of continuous insulin infusion in noncritical care settings. J Hosp Med 2010;5:212-17. https://doi.org/10.1002/jhm.646 